\title{
Nonlinear Interaction and Reflection of Incoherent Light Beams
}

\author{
D. V. Gorbach ${ }^{a}$, O. G. Romanov ${ }^{a}$, A. P. Sukhorukov ${ }^{b}$, and A. L. Tolstik ${ }^{a}$ \\ ${ }^{a}$ Belarusian State University, Nezalezhnasti av. 4, Minsk, 220030 Belarus \\ ${ }^{b}$ Moscow State University, Moscow, 119991 Russia \\ e-mail: gorbach@tut.by; romanov@bsu.by; apsmsu@gmail.com
}

\begin{abstract}
The interaction dynamics between two incoherent light beams in a medium with thermal nonlinearity is studied both experimentally and theoretically. It is shown that in the mode of a nonlinear reflection the transformation of a spatial structure of a weak signal beam can be theoretically described in the framework of a spatially distributed model for a nonlinear change in the refractive index of the medium.
\end{abstract}

DOI: $10.3103 / \mathrm{S} 1062873810120014$

\section{INTRODUCTION}

The possibilities for controlling the parameters of light beams in the mode of nonlinear interactions have been discussed intensively in the scientific literature [1-4]. The interest in studies of the systems based on the principle of controlling light by means of light is due to the potential possibilities of using the advantages of optical methods of processing information. Fast-response switching devices can be obtained, for example, in media with quadratic [5] or thermal [6] nonlinearities. The sphere of practical application can thus be enlarged by using, along with soliton light beams, a nonsolitonic mode for the distribution and interaction of beams.

Peculiarities of the incoherent interaction of the pulse light beams in the media with a thermal nonlinearity are analyzed both experimentally and theoretically in this work. Solutions of complex organic dyes that allow us to work with high-power nanosecond laser pulses were selected as the nonlinear medium. Peculiarities of the transformation of the spatial structure of the light beams and the conditions under which a nonlinear version of a total internal reflection upon the interaction of beams in a defocusing medium can be attained are discussed.

\section{EXPERIMENTAL}

The experimental attainment of the effect of a nonlinear reflection in a medium with nonlinearity is based on the creation of a localized spatial region with a negative change in the refractive index. Such a region can be formed in a solution of organic dye with a negative thermo-optic coefficient $(d n / d T<0)$ under the effect of a high-power laser pulse (a pump beam). The propagation of a laser pulse in a solution of a dye creates an inhomogeneous distribution of temperature in a cross section of a nonlinear medium. As a result, a gradient of the refractive index is produced, and a high-power reference beam becomes a nontransparent object for an oblique wave of different frequency. Under certain conditions, we can detect on a lightinduced phase inhomogeneity the dissipation of a signal beam propagating at a small angle to the pump beam.

For experimental realization of the mode of nonlinear reflection, the continuous radiation of a helium-neon laser with a wavelength of $\lambda=632.8 \mathrm{~nm}$ was used as the signal beam. The yttrium aluminum garnet laser generated a high-power light pulse with a wavelength of $\lambda=1064 \mathrm{~nm}$. The laser was operating in the mode of active $Q$-modulation (with a $20 \mathrm{~ns}$ pulse duration, a $1.5 \mathrm{mrad}$ laser radiation divergence, and at a $1-10 \mathrm{~Hz}$ pulse repetition frequency). A system of movable mirrors allowed us to change the angle between the light beams over the range of 10-100 mrad. Long-focus lenses $(30-60 \mathrm{~cm})$ were used to increase the intensity of pumping. An ethanol solution of $3274 U$ polymethine dye, which absorbs radiation at the wavelength of a pump pulse laser's generation $(\lambda=$ $1064 \mathrm{~nm}$ ), and which was actually transparent in the visible region of a spectrum, was used as the medium with a thermal nonlinearity. The rapid and efficient thermalization of the adsorbed energy resulted from a low quantum yield of luminescence (below 10\%) and the short lifetime of the polymethine dye molecules in the excited state $\left(\sim 10^{-10}-10^{-11} \mathrm{~s}\right)$. The resulting picture of interaction was registered on a screen using CCD camera. In the course of our experimental research, the peculiarities of the propagation (passage and reflection) of the signal light beam (the radiation of a helium-neon laser) through the light-induced region formed in the dye solution by the high-power focused radiation of a laser on an yttrium aluminum garnet were analyzed. 
(a)
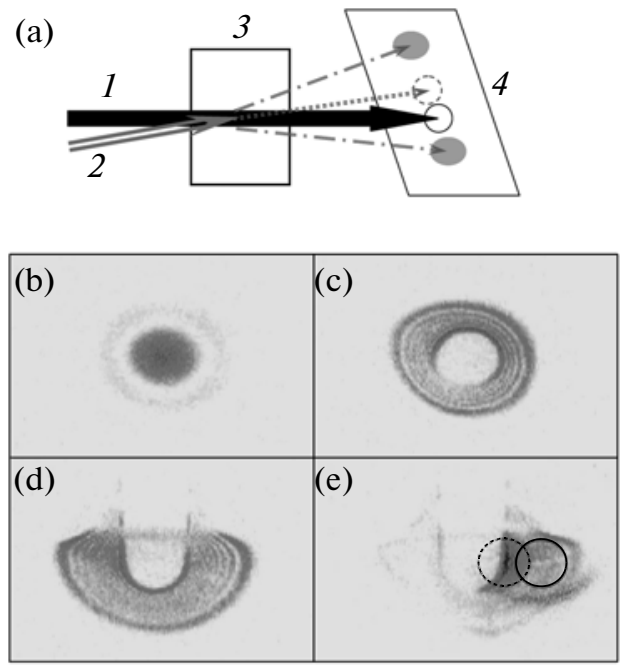

Fig. 1. Scheme of the nonlinear interaction of a light beam (a): 1 pump beam, 2 signal beam, 3 nonlinear medium, 4 screen. Distribution of the intensity of a signal beam transmitted through a high-power pump beam at a number of excitation laser pulses: $N=0$ (b), 6 (c), 12 (d) and upon the partial reflection of the signal beam (e).

In accordance with our theoretical estimation, the maximum value of the angle between the signal beam and pump beam $\gamma$ at which a total internal reflection is realized is determined by a nonlinear change in the refractive index: $\gamma \approx \sqrt{|\Delta n|}$. In the case under study, the change of the refractive index $\Delta n$ is correlated with a thermal nonlinearity whose value can be estimated using the following formula: $\Delta n=\frac{d n}{d T} \frac{k_{a} I \tau}{C_{\rho}}$, where $d n / d T$ is the thermo-optic coefficient; $k_{a}$ denotes the absorption coefficient of the dye solution at the pump frequency; $I$ implies the peak intensity of the pump pulse; $C_{\rho}$ designates the thermal capacity of a unit of volume; and $\tau$ is the effective time of interaction, which coincides with the pulse duration of $\tau_{p}$ during operation with single pulses, and of $\tau \approx N \tau_{p}$ when the laser is operating in a frequency mode where $N$ is the number of pulses at which the thermal processes become stationary.

Figure 1a shows a diagram of the nonlinear interaction between the radiation of a helium-neon laser and a pump beam. Pump beam 1 and signal beam 2 were directed into a cell with a dye solution 3 . The form of the signal beam after the interaction was registered on screen 4. At an intensity of the pump beam of $I=$ $100 \mathrm{MW} \mathrm{cm}^{-2}$ which propagated in the ethanol dye solution (a thermo-optic coefficient $d n / d T=-4 \times$ $10^{-4} \mathrm{~K}^{-1}$, a thermal capacity of a unit of volume $C_{\rho}=$ $2 \mathrm{~J} \mathrm{~cm}^{-3} \mathrm{~K}^{-1}$, and an absorption coefficient $k_{a}=1 \mathrm{~cm}^{-1}$ ), a threshold angle between the interacting beams was $\gamma=20 \mathrm{mrad})$. A dashed line and a circle in Fig. 1a show the direction of propagation and location of the signal beam in the absence of radiation of the pump beam. Switching on the oscillation of the laser on yttrium aluminum garnet induced the change of direction of propagation of the signal beam, which is exhibited schematically by the dash-and-dot arrows. The fixed distributions of the intensity in the profile of the signal beam (Fig. 1), show the dynamics of transformation of radiation of the helium-neon laser depending on a quantity of the pulses of the high-power beam, provided the maximum overlapping of a signal beam and a pump beam occurs in the dye cell. In the absence of a pump beam, a Gaussian beam of radiation of the helium-neon laser can be seen at the output (Fig. 1b). Owning to a local thermal growth in the medium generated by the laser on yttrium aluminum garnet, a region with a lower value of the refractive index $(\Delta n<0)$ is created, which starts extruding a signal beam off the center (Fig. 1c). However, in due course, ( $\tau \sim 1 \mathrm{~s}$ of a laser operating at $10 \mathrm{~Hz}$ frequency) resulting from the convection flows in the dye solution, a symmetry is broken and the upward removal of heat from a related region of a thermal change of the refractive index, occurs. In this case, a vertical heat wall forms which participates in the process of a nonlinear reflection (Fig. 1d).

On a displacement of a signal beam in a horizontal plane from a position of a total overlapping, the character of the interaction changes: a partial repulsion of a probing beam is observed (Fig. 1e). The initial direction of propagation of the signal beam radiation (the dashed circle) is transformed into the area of the angles within the range from the initial propagation to a direction of total reflection of the signal beam from the pump beam. The solid circle in the figure is a position of a high-power IR pump beam.

To realize a total reflection of a beam from the region of the light-induced nonlinearity, an intensity of the pump beam was increased to $I=1.5 \mathrm{GW} \mathrm{cm}^{-2}$, and, the angle between the interacting beams was decreased to $\gamma=10 \mathrm{mrad}$. Under such conditions, the reflection of the signal beam occurred resulting from the effect of the single radiation beams on the medium, with no formation of a heat wall. A dynamics of interaction of the beams is shown in Fig. 2. Occurrence of a high-power pulse in the cell with the dye solution involved a sharp displacement of the signal beam radiation from the initial position (Fig. 2a) to the region corresponding to the direction of a total reflection of a beam from the thermally-induced region (Fig. 2b). Parallel to heat redistribution in bulk of the medium, a decrease of the value of a heat change of the refractive index took place, and radiation of the signal beam was approaching its initial position (Fig. 2c, 2d). The initial size of the signal beam were attained in 150-200 ms, and the final relaxation of the thermally-induced region and the signal beam's return to its initial direction of propagation lasted one second. 
A comparison of the two modes of interaction (the intensity $I=100 \mathrm{MW} \mathrm{cm}^{-2}$ at a pulse repetition of $10 \mathrm{~Hz}$ and the intensity of $I=1.5 \mathrm{GW} \mathrm{cm}^{-2}$ for single pulses) shows that with single laser pulses, the signal beam is transformed into a region of the angles that satisfy the condition of total internal reflection. Under the conditions of frequency excitation, a large-aperture dissipation of the signal beam is also observed that could be correlated with the transformation of the thermally-induced region owing to the manifestation of the convection effect and that of heat relaxation.

\section{THEORETICAL MODEL}

To explain the dynamics of the transformation of the transverse structure of a signal light beam upon its reflection from the spatial-inhomogeneous thermal lens generated in the dye solution by the pump beam, a theoretical model was developed that considers both the dynamics of the change in the refractive index of the medium under the effect of a high-power light pulse and the volumetric character of the interaction of the light beams.

To describe theoretically the process of the formation of the spatial-inhomogeneous structures of the refraction index, assume that a high-power pump wave $E_{1}$ propagating in a nonlinear medium is characterized by an optical frequency $\omega$ that coincides with the center of an absorption line $S_{0}-S_{1}$ of the dye solution. The probe wave $E_{S}$ at a frequency $\omega^{\prime}$ for which the medium is transparent propagates at a narrow angle $\gamma$ to the pump wave. For the dye solutions in our theoretical modeling, the key role in forming the optic response upon monopulse and frequency excitation is considered to belong to the resonance and heat nonlinearity.

Under these conditions, the expressions for the nonlinear susceptibility of the medium at frequencies $\omega$ and $\omega^{\prime}$ are

$$
\begin{gathered}
\chi(\omega)=\frac{n_{0} k_{0}}{2 \pi}\left(\frac{\hat{\Theta}_{12}}{B_{12}}-\frac{\hat{\alpha} I_{1}}{1+\alpha I_{1}}\right), \\
\chi\left(\omega^{\prime}\right)=\frac{n_{0} \kappa_{0}}{2 \pi} \frac{a_{T} I_{1}}{1+\alpha I_{1}},
\end{gathered}
$$

where $\quad \hat{\alpha}=a+i \alpha=\left(\hat{\Theta}_{12}+\hat{\Theta}_{21}\right) / v P_{12}-a_{T}, \quad a_{T}=$ $2 \omega(d n / d T)\left(1-\mu_{21}\right) \tau / c C_{\rho}$. In the given expressions $\hat{\Theta}_{k l}(\omega)=\Theta_{k l}(\omega)+i B_{k l}(\omega)$ where the coefficients $\Theta_{k l}(\omega)$ are associated with the Einstein coefficients by the Kramers-Kronig ratios for stimulated transitions $B_{k l}(\omega)$ in the spectral channel $S_{0}-S_{1}, v$ is the speed of light in the medium, $\kappa_{0}$ is the initial coefficient of extinction, $n_{0}$ signifies the refractive index of the solvent, $P_{21}$ denotes the total probability of spontaneous and nonradiative transitions, $\mu_{21}$ is the quantum yield

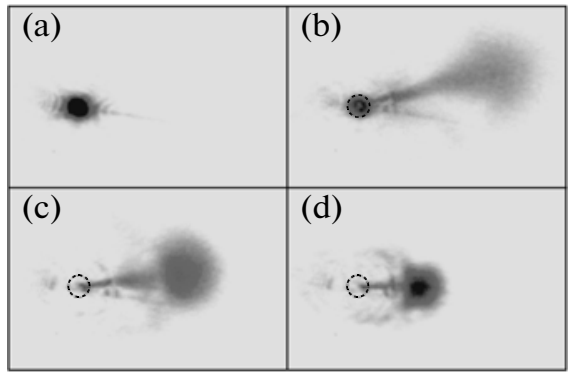

Fig. 2. Dynamics of the transformation of the signal beam upon reflection from a high-power pulse pump beam at various times of observation $t=0$ (a), $0-30 \mathrm{~ms}(\mathrm{~b}), 30$ $60 \mathrm{~ms}$ (c), 60-120 ms (d).

of luminescence in the channel $S_{0}-S_{1}$, and $I_{1}$ denotes the intensity of the pump wave.

The dynamics of the spatial change of the refractive index of the medium $\Delta n=\frac{d n}{d T} \Delta T$ is determined by its local heating $\Delta T(x, y, z, t)$ in the field of the pump wave, which can be found from the equations of heat conduction

$$
C_{\rho} \frac{\partial T}{\partial t}=\delta\left(\frac{\partial^{2} T}{\partial x^{2}}+\frac{\partial^{2} T}{\partial y^{2}}+\frac{\partial^{2} T}{\partial z^{2}}\right)+Q_{s},
$$

where the function of heat release is defined by the geometrical and time parameters of the pump wave $Q_{s}=k_{a} I_{1}(x, y, z, t), k_{a}$ is the absorption coefficient of the dye solution at a pump frequency, and $\delta$ denotes the coefficient of heat conduction of the solvent.

The equations for the complex amplitudes of light waves $E_{1}$ and $E_{S}$ with allowance for the nonlinear modulation of the absorption coefficient and the refractive index in the field of a high-power pump wave can be written as

$$
\begin{gathered}
\frac{\partial E_{1}}{\partial z}+\frac{1}{2 i k_{1}} \Delta_{\perp} E_{1}=\frac{i 2 \pi \omega}{c n_{0}} \chi(\omega) E_{1} ; \\
\frac{\partial E_{S}}{\partial z}+\gamma \frac{\partial E_{S}}{\partial x}+\frac{1}{2 i k_{S}} \Delta_{\perp} E_{S}=\frac{i 2 \pi \omega^{\prime}}{c n_{0}} \chi\left(\omega^{\prime}\right) E_{S},
\end{gathered}
$$

where $k_{1}=\omega^{\prime} n_{0} / c$ and $k_{S}=2 \omega^{\prime} n_{0} / c ; \Delta_{\perp}=\partial^{2} / \partial x^{2}+$ $\partial^{2} / \partial y^{2}$ is a transverse Laplacian.

Considering an obvious form of the expressions for a nonlinear susceptibility $\chi(\omega)(1)$ and $\chi\left(\omega^{\prime}\right)(2)$, the system of coupled wave equations (4) is transformed into the following form

$$
\begin{gathered}
\frac{\partial E_{1}}{\partial \zeta}=i \Delta_{\perp}^{\prime} E_{1}+i k_{a} L_{D}\left(\frac{\hat{\Theta}_{12}}{B_{12}}-\frac{\hat{\alpha} I_{1}}{1+\alpha I_{1}}\right) E_{1}, \\
\frac{\partial E_{S}}{\partial \zeta}+\gamma^{\prime} \frac{\partial E_{S}}{\partial \xi}=i \Delta_{\perp}^{\prime} E_{S}+2 i k_{a} L_{D}\left(\frac{a_{T} I_{1}}{1+\alpha I_{1}}\right) E_{S},
\end{gathered}
$$

where $\Delta_{\perp}^{\prime}=\partial^{2} / \partial \xi^{2}+\partial^{2} / \partial \eta^{2}, \gamma^{\prime}=2 \gamma L_{D} / r_{0}$ : the spatial coordinates are normalized as follows: $\xi=x / r_{0}$, 


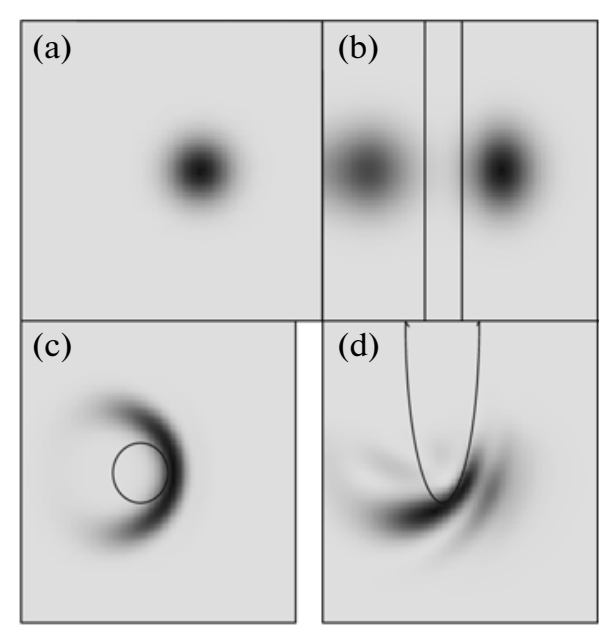

Fig. 3. Spatial profile of a probe light beam after passing through an induced inhomogeneity with a refractive index of different form.

$\eta=y / r_{0}$, and $\zeta=z / 2 L_{D}$. The diffraction length $L_{D}=2 \pi r_{0}^{2} / \lambda$ is determined by the width of the pump beam $r_{0}$.

In the numerical modeling of equation systems (3) and (5), the light pump beam directed onto the boundary $z=0$ of a nonlinear medium was assumed to have the Gaussian profile with respect to transverse coordinates: $I_{1}(z=0, x, y)=I_{10} \exp \left[-\left(x^{2}+y^{2}\right) / r_{0}^{2}\right]$. The half-width of the light beam upon entering a nonlinear medium was assumed to be $r_{0}=100 \mu \mathrm{m}$, the peak beam intensity varied within the range of $I_{1}=$ $0.1-1 \mathrm{GW} \mathrm{cm}^{-2}$, the wavelength of radiation was $\lambda_{1}=$ $1 \mu \mathrm{m}$, the duration of the laser pulse was $\tau=20 \mathrm{~ns}$, and the initial absorption coefficient was selected to be on the order of $k_{a}=1 \mathrm{~cm}^{-1}$. The probe light beam with a wavelength of $\lambda_{S}=0.63 \mu \mathrm{m}$ was also characterized by a Gaussian-like intensity distribution in the cross section: $I_{S}(z=0, x, y)=I_{S 0} \exp \left[-\left(\left(x-x_{0}\right)^{2}+(y-\right.\right.$ $\left.\left.\left.y_{0}\right)^{2}\right) / r_{0}^{2}\right]$ and was directed into a nonlinear medium at a narrow angle of $\gamma \approx 20 \mathrm{mrad}$. The distance between the centers of the light beams at the boundary $z=0$ was $x_{0} \approx r_{0}$. The following spectral and thermo-optic characteristics typical for the $3274 \mathrm{U}$ dye solution in ethanol were used for the medium3274U: $n_{0}=1.36$, the quantum yield of luminescence was $\mu_{12}=0.01$, and the thermo-optic parameter was $d n / d T\left(C_{\rho}\right)^{-1}=-2 \times 10^{-4} \mathrm{~J}^{-1} \mathrm{~cm}^{3}$.

Figure 3 shows the results from our numerical modeling in the form of the intensity distributions of a probe light beam in a transverse profile $I_{S}(x, y)$ upon exiting the nonlinear medium $z=L$ for three different geometries of a spatial-inhomogeneous profile $\Delta n(x, y, z)$.

Equation (3) tells us that upon the absorption of the pump radiation in a nonlinear medium, a negative thermal lens is induced whose geometry is determined by the spatial distribution of intensity in the pump beam. In the first of the reported cases, an initial probe beam (Fig. 3a) was directed into a nonlinear medium to fulfill the conditions of total internal reflection. It was partially reflected with a less than complete transition (Fig. 3b) through the inhomogeneity in the form of a wall having a thickness of $\Delta x \approx r_{0}$ (the solid lines in Fig. 3b). As we can see from the results of our numerical modeling, the coefficient of reflection from an inhomogeneity of this type depends substantially on the thickness of the wall $\Delta x$ and tends to unity at $\Delta x \gg r_{0}$.

The second variant of interaction (Fig. 3c) is relevant to the experimental conditions (Fig. 1c) and demonstrates the regime of the deformation of the intensity distribution in a transverse profile of the signal beam, a result of the transition through the cylindrical inhomogeneity created in the bulk of the medium by the Gaussian pump beam.

An appropriate profile of an inhomogeneity in the refractive index (the solid line in Fig. 3 d) was selected to model the interaction of light beams under conditions of formation of a vertical heat wall. It thus can be seen from this figure that the distribution of the intensity upon exiting a nonlinear medium qualitatively coincides with that registered experimentally using the frequency mode of succession of the pump pulses (Fig. 1e).

\section{CONCLUSIONS}

Our theoretical and experimental analyses of the interaction and reflection of incoherent laser beams in a medium with thermal nonlinearity allowed us to determine the conditions under which the effect of nonlinear reflection occurs. The preferred transformation of a signal beam into a region of angles that fit the geometrical reflection of the signal beam from the pump beam was demonstrated.

\section{ACKNOWLEDGMENTS}

This study was supported by the Republic of Belarus Foundation for Basic Research, project F10R-070, and by the Russian Foundation for Basic Research, project nos. 10-02-90010-Bel_a and 09-0201028-a. 


\section{REFERENCES}

1. Gibbs, H.M., Optical Bistability: Controlling Light by Light, Orlando: Acad. Press, 1985.

2. Rozanov, N.N., Opticheskaya bistabil'nost' i gisterezis $v$ raspredelennykh nelineinykh sistemakh (Optical Bistability and Hysteresis in Distributed Nonlinear Systems), Moscow: Nauka, 1997.

3. Kivshar', Yu.S. and Agraval, G.P., Opticheskie solitony. Ot voloknistykh svetovodov $k$ fotonnym kristallam (Opti- cal Solitons. From Fiber Light Guides to Photon Crystals), Moscow: Fizmatlit, 2005.

4. Dissipativnye solitony (Dissipative Solitons), Akhmadiev, N. and Ankevich, A., Eds., Moscow: Fizmatlit, 2003.

5. Lobanov, V.E. and Sukhorukov, A.P., Izv. Akad. Nauk. Ser. Fiz., 2005, vol. 69, no. 12, p. 1755.

6. Lobanov, V.E., Kalinovich, A.A., Sukhorukov, A.P., et al., Laser Phys., 2009, vol. 19, p. 1112. 